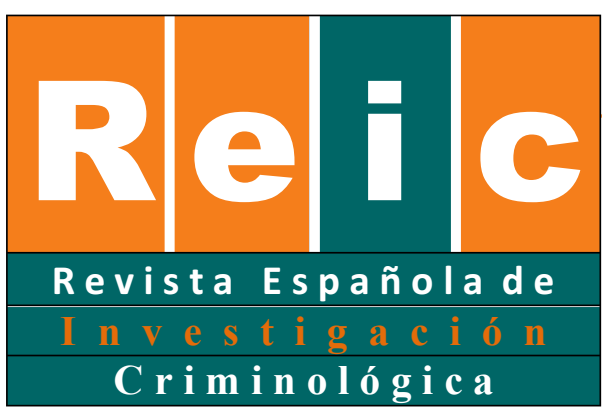

\title{
Comunidad y delincuencia juvenil: El rol mediador de la familia y el grupo de iguales
}

\section{Community and delinquency: The mediating role of family and peer group}

Artículo recibido el 30 de marzo 2016/Publicado el 16 de septiembre de 2016

\author{
Juan Antonio Rodríguez ${ }^{1}$ \\ Universidad de Los Andes
}

\begin{abstract}
RESUMEN
Este estudio explora el papel mediador de la conducta parental y el grupo de amigos desviados en la relación entre comunidad y delincuencia juvenil. Para ello, se analiza una muestra venezolana de 1011 adolescentes escolarizados de 11 a 19 años de edad. Los resultados derivados de un Análisis de Estructuras de Covarianzas indican que la relación del desorden social y la eficacia colectiva con la delincuencia juvenil está mediada en parte por la conducta parental y el grupo de iguales desviados.
\end{abstract}

Palabras clave: Comunidad, familia, grupo de iguales, delincuencia juvenil

\begin{abstract}
This study explores the mediating role of parental behaviour and deviant peer group in the relationship between the community and delinquency. A sample of 1,011 Venezuelan adolescent students, ranging from ages 11 to 19 years old was analysed. The results derived from the Analysis of Covariance Structures indicate that the relationship between social disorder and collective efficacy with delinquency is mediated in part by parental behaviour and a deviant peer group.

\footnotetext{
${ }^{1}$ La correspondencia debe dirigirse a Juan Antonio Rodríguez. Av. Las Américas, Campus LIRIA, Facultad de Ciencias Jurídicas y Políticas, Escuela de Criminología, C.P. 5101. Mérida. Venezuela. jarodrig@ula.ve
} 
Key words: Community, family, peer group, delinquency

\section{Introducción}

La comunidad es un ámbito fundamental en el desarrollo de los adolescentes, puesto que brinda oportunidades esenciales para la conformación de redes de apoyo social (Pretty, 2002). Sobre esto, la investigación ha establecido que los jóvenes que perciben en sus lugares de residencia más oportunidades de relacionarse con otros, cohesión entre los vecinos y apoyo social de los amigos, manifiestan mayores niveles de conducta prosocial (Lenzi et al., 2011). Sin embargo, no siempre este contexto de socialización es fuente de efectos positivos en la conducta de niños y adolescentes. Desde el primer tercio del siglo pasado, un número significativo de estudios tanto en Sociología como en Criminología ha evidenciado el impacto de la comunidad en la delincuencia juvenil (Downes y Rock, 2007; Leventhal y Brooks-Gunn, 2000).

En tal sentido, la producción teórico-empírica coincide en que las tasas de delincuencia juvenil varían de forma sistemática entre áreas residenciales y esto guarda relación con las propias características del medio (Chung y Steinberg, 2006; Sampson, 2004; Sampson, Raudenbush y Earls, 1997; Sampson y Groves, 1989; Shaw y McKay, 1942; Wilson y Kelling, 1982). Los investigadores han aislado dos tipos de dimensiones a nivel comunitario de gran interés para la reflexión criminológica y que, según sus resultados, se encuentran estrechamente vinculadas (Mrug y Windle, 2009; Sampson et al., 1997). Una de ellas está representada por las características estructurales de la comunidad (v. gr. pobreza, clase social, desempleo, movilidad residencial, etc.) y la otra por los procesos sociales que en ésta se desarrollan (v. gr. lazos vecinales, vinculaciones sociales, control social informal, desorden, etc.) (Leventhal y Brooks-Gunn, 2000; Mrug y Windle, 2009). En el marco de lo anterior, los investigadores realizan una discusión muy valiosa sobre la manera en cómo las características tanto estructurales como sociales de la comunidad se asocian con la delincuencia juvenil (Leventhal y BrooksGunn, 2000). Una de las líneas de análisis propugna que el ámbito comunitario se relaciona con el comportamiento desadaptado de los jóvenes por medio del funcionamiento de otros entornos de socialización más inmediatos como la familia y los 
amigos (v. gr. Chung y Steinberg, 2006; Rankin y Quane, 2002). Con base en los estudios internacionales, el propósito principal de este trabajo es analizar en una muestra de adolescentes venezolanos, si el desorden social y la eficacia colectiva presentan como procesos sociales comunitarios una relación indirecta con la delincuencia juvenil mediante las prácticas parentales y el grupo de amigos desviados.

\section{Marco teórico}

\subsection{Comunidad y delincuencia juvenil: efectos mediadores en la perspectiva de la desorganización social}

Desde principios del siglo pasado, varios investigadores han identificado que los problemas de ajuste en la adolescencia guardan relación con algunos contextos sociales como la comunidad, la familia y el grupo de iguales (Haynie, Silver y Teasdale, 2006). Por ejemplo, los trabajos de Shaw y McKay (1942) señalan que las comunidades en transición de una ciudad en plena industrialización en las que, por lo general, hay altas tasas de delincuencia, tienen características o factores estructurales muy particulares: alta rotación poblacional, pobreza y diversidad étnica. Al parecer estas condiciones comunitarias no son directamente responsables del delito; en su lugar, actúan en el mediante la desorganización social. Se trata por ende de que estas desventajas estructurales se asocian tanto con la carencia de prácticas familiares adecuadas como con las dificultades de integración y control social por parte de los vecinos lo cual tiende a aumentar la delincuencia juvenil.

Con base en estas ideas, Sampson y Groves (1989) sostienen posteriormente que algunas características estructurales de comunidades desfavorecidas (v. gr. bajo nivel económico, movilidad residencial, desintegración familiar, etc.) son fuentes exógenas de desorganización social que, por intermedio de ésta, conllevan al débil funcionamiento de las instituciones sociales locales encargadas de controlar la desviación. Precisamente este modelo explicativo supera las propuestas de Shaw y McKay porque ofrece una definición expresa de lo que significa desorganización social. En sí, este proceso social representa la incapacidad de la comunidad para hacer efectivos los valores comunes de sus residentes y para mantener un eficaz control sobre 
sus comportamientos (Sampson y Groves, 1989: 777). Según las predicciones teóricas de Sampson y Groves, la desorganización social se caracteriza por débiles redes de amistad en el vecindario, poco capital social, baja participación organizacional e insuficiente comunicación entre los vecinos. La fragilidad de los lazos sociales condiciona la capacidad de las comunidades para desarrollar redes en las que se inserta la familia, capaces de regular, supervisar y corregir la delincuencia de los jóvenes.

Más adelante, Sampson, Reudenbush y Earls (1997) propugnan que los factores estructurales de algunas localidades afectan tanto a la delincuencia como al desorden social y físico por medio de una forma de capital social que denominan 'eficacia colectiva'. La eficacia colectiva se refiere al rol que juegan la confianza mutua, la solidaridad, las redes y las normas a favor del bienestar común en el ámbito vecinal. Así, la eficacia colectiva está estructurada por dos factores: la cohesión social y el control social informal e implica la posibilidad de alcanzar de modo mancomunado ciertos objetivos del vecindario tales como seguridad, ornato público, educación y salud (Sampson, 2004). Sampson (2004) señala que las respuestas a la inseguridad y al desorden social/físico aumentan cuando los vecinos construyen lazos comunitarios estables y, más importante aún, fiables. Es poco probable que los vecinos promuevan el control social y el orden público o, inclusive, que logren cualquier otro propósito colectivo si la cohesión social es frágil. De tal manera, la interacción entre los residentes y la confianza en la intervención organizada, sumada a la disposición de ejercer supervisión y cuidado sobre los jóvenes, son procesos sociales básicos que favorecen la socialización en la comunidad y previenen la delincuencia juvenil. Cabe resaltar, especialmente, que en este proceso de influencias los escasos niveles de eficacia colectiva favorecen la poca supervisión parental, pero también la delincuencia que experimentan los jóvenes en sus grupos de iguales porque los vecinos no procuran contener este tipo de comportamientos.

Un aporte fundamental que resume lo visto anteriormente es que las características estructurales de las comunidades menos favorecidas impiden procesos sociales que promuevan el comportamiento convencional de los vecinos, la familia y los jóvenes. La perspectiva de la desorganización social resulta primordial porque plantea la idea de efectos distales entre la comunidad y la delincuencia juvenil y, además, estimula 
la discusión del posible rol que pueden jugar en esta relación los agentes de socialización primaria, esto es, familia y amigos.

\subsection{Conducta parental, grupo de amigos antisociales y delincuencia juvenil}

La Criminología ha desarrollado una base teórico-empírica que respalda un conjunto de argumentos sobre relaciones directas, indirectas e, incluso, bidireccionales entre variables referidas al funcionamiento familiar, amigos desviados y delincuencia juvenil (v. gr. Chung y Steinberg, 2006; de Kemp, Scholte, Overbeek y Engels, 2006; Dishion, Bulluck y Granic, 2002; Gottfredson y Hirschi, 1990; Hoeve et al., 2009; Jessor y Jessor, 1977; Mirón y Otero-López, 2005; Rodríguez, Mirón y Rial, 2012; Scaramella, Conger, Spoth y Simons, 2002). Con referencia a esto, los problemas de crianza se han asociado a la desviación de los hijos, pero también a la toma de contacto de ellos con amigos delincuentes. El grupo de iguales antisociales representa uno de los instigadores más fuertes de la delincuencia juvenil, cuya influencia es explicada regularmente por mecanismos de aprendizaje y/o de presión grupal (Mirón y Otero-López, 2005; Patterson, Dishion y Yoerger, 2000). La investigación ha revelado que la implicación con amigos desviados puede contribuir substancialmente a explicar los efectos de las prácticas parentales en la delincuencia juvenil (Deutsch, Crockett, Wolff y Russell, 2012; Mirón y Otero-López, 2005).

Acerca de esto, el abordaje sistemático de la relación padres y conducta antisocial de los hijos ha analizado en particular dos dimensiones: a) la afectividad y b) el control (normas y supervisión) (Hoeve et al., 2009). En cuanto a la afectividad, numerosas investigaciones sugieren que la calidad de las interacciones afectivas entre padres e hijos en el transcurso de la niñez y la adolescencia (v. gr. apoyo, cohesión, comunicación, etc.) presentan de forma directa efectos beneficiosos en el ajuste y desarrollo psicosocial del joven, en particular, porque disminuyen la probabilidad de delincuencia (de Kemp et al., 2006; Gorman-Smith, Tolan y Henry, 2000; Mirón y Otero-López, 2005). En cambio, la falta de apoyo y vinculación familiar está asociada a distintos problemas del adolescente y puede generar en él dificultades personales importantes entre las cuales se especifica cierta susceptibilidad al contacto con amigos desviados y la tendencia a la conducta antisocial (Rodríguez, Mirón y Rial, 2012; 
Rodríguez, 2011; Mirón y Otero-López, 2005; Scaramella et al., 2002). Sobre esto, en varios modelos explicativos como, por ejemplo, el de Jessor y Jessor (1977), se proponen mecanismos mediante los cuales la calidad de la relación familiar puede incidir en la afiliación de los hijos a grupos de iguales desviados.

La segunda dimensión, aquella relativa al control parental, se desdobla en dos elementos esenciales dentro de la socialización (Hoeve et al., 2009). El primero se refiere a las normas que los padres intentan que sus hijos internalicen con el objetivo de que los adolescentes respeten las reglas y convenciones para la mejor convivencia social. Y el segundo aspecto asocia el control familiar con el concepto de supervisión, indicando el grado de vigilancia e inspección de los padres sobre sus hijos, vale decir, la cantidad y calidad de información que los progenitores tienen de la vida diaria de los adolescentes, en especial, cuando están fuera del hogar. La supervisión familiar constituye de forma directa un factor protector con respecto a la delincuencia juvenil (de Kemp et al., 2006) y de modo indirecto también consigue reducirla por medio de su efecto en la selección o influencia del grupo de amigos desviados (Rodríguez et al., 2012; Rodríguez, 2011; Mirón y Otero-López, 2005). De manera general, esta línea de investigación apunta a que el apoyo y la supervisión familiar promueven un fuerte vínculo entre padres e hijos, el cual puede amortiguar tanto la incidencia de los amigos desviados como la propia delincuencia del adolescente (Mirón y Otero-López, 2005).

\subsection{Comunidad, familia, grupo de iguales y delincuencia juvenil: hallazgos sobre efectos de mediación}

Con base en algunas teorías ecológicas, varios trabajos han incorporado al análisis de las variables estructurales otros atributos referidos a procesos sociales muy particulares que se desarrollan en la comunidad, que unidos a variables familiares y grupales, actúan en la desviación juvenil. Uno de ellos comprueba que bajos niveles de eficacia colectiva en el vecindario predicen la inadecuada supervisión parental, una mayor probabilidad de involucrarse con amigos desviados y altos niveles de delincuencia juvenil y consumo de drogas (Rankin y Quane, 2002). Estos investigadores informan que la relación entre eficacia colectiva y delincuencia juvenil es sólo indirecta y en ella se interponen las prácticas familiares y el grupo de iguales. En el estudio de Tolan, Gorman-Smith y 
Henry (2003) se sostiene que tanto la conducta parental como la implicación de los adolescentes con amigos desviados son mediadoras de los efectos de la comunidad en los delitos violentos. En concreto, esta investigación establece que la debilidad de las características estructurales y el desorden social están indirectamente vinculados a la participación en grupos desviados mediante el efecto en las prácticas parentales (baja supervisión, disciplina dura y baja participación familiar) y que tener amigos desviados media la influencia de la conducta parental ineficaz en la violencia del adolescente.

Chung y Steinberg (2006) desarrollan un modelo hipotético de la delincuencia grave en el cual plantean algunos efectos directos e indirectos entre los factores estructurales (v. gr. concentración de la pobreza, diversidad étnica e inestabilidad residencial) y sociales (v. gr. desorden social/físico y cohesión social) de la comunidad, la conducta parental y el contacto con amigos desviados. A este respecto, en dicho modelo destacan principalmente las relaciones indirectas de las variables comunitarias con la delincuencia juvenil por medio del desempeño familiar y el contacto con grupo de iguales desviados. Los resultados van en la misma línea de los estudios anteriores: la supervisión y el afecto de los padres se asocian de forma negativa con la delincuencia grave (homicidio, prostitución y robo a mano armada), y tener iguales desviados, además de percibir desorden social y físico en el vecindario, aumenta la probabilidad de delincuencia juvenil. Al mismo tiempo, se comprueba el efecto indirecto del desorden y la cohesión social en la delincuencia grave, mediante la relación directa de estas variables con la conducta parental y los amigos desviados. En esta misma dirección, Mrug y Windle (2009) han puesto a prueba un modelo de mediación similar al de Chung y Steinberg para explicar la conducta externalizante de jóvenes preadolescentes. Los principales hallazgos de este estudio señalan que algunos indicadores como la pobreza tienen un efecto en el comportamiento problemático del joven y esta relación está mediada por el desorden social, la cohesión en la comunidad y la pobre gestión parental. Sin embargo, a diferencias de Chung y Steinberg estos autores no contrastan el papel mediador del grupo de amigos desviados.

Por otra parte, algunas investigaciones recientes han puesto a prueba modelos de mediación para explicar los efectos de la comunidad sobre la conducta de los adolescentes en los que se descartan de las hipótesis de partida cualquier variable de tipo estructural (v. gr. Byrnes, Miller, Chen y Grube, 2011; Vieno, Nation, Perkins, 
Pastore y Santinello 2010). En el estudio de Vieno y su equipo, los principales hallazgos indican que bajos niveles de capital social (v. gr. bajo apoyo de los vecinos y mal clima social) se asocian con mayor miedo al delito de los padres y, también, con escaso apoyo y supervisión parental basada en la solicitud de información sobre actividades sin vigilancia directa de los hijos. La percepción de problemas de seguridad en los entornos residenciales incide en los procesos de crianza de los padres. De manera general, este estudio ratifica que los procesos sociales de la comunidad afectan el comportamiento del adolescente mediante la crianza familiar. Finalmente, Byrnes et al. (2011) señalan que las madres que perciben problemas en su comunidad (incluidos la delincuencia y el desorden social y físico) gestionan más estrategias de supervisión basadas en el conocimiento del lugar en el que se encuentran sus hijos. Esta forma de gestión parental afecta de modo directo la interacción de los hijos con amigos consumidores de sustancias, e indirectamente la implicación en delincuencia y consumo de alcohol.

\subsection{El presente estudio}

El propósito principal de este estudio es entender las posibles vías por las cuales se vinculan el entorno residencial y la delincuencia juvenil en una muestra de adolescentes venezolanos. Dado que este es un tema poco tratado en el contexto latinoamericano, cabe preguntarse si la relación entre la percepción de desorden social y eficacia colectiva con la delincuencia autoinformada de adolescentes venezolanos se da por medio de la conducta parental y el grupo de amigos y, por ende, hay una influencia indirecta. Ofrecer un intento de respuesta a esta pregunta en el contexto venezolano, puede robustecer el conocimiento acerca de un patrón de hipótesis muy discutido que defiende los efectos mediadores en la relación comunidad y delincuencia juvenil.

En la Figura 1 se detalla el modelo conceptual que se asume en este trabajo para intentar responder a la pregunta de investigación. En este modelo se establece un conjunto de relaciones que pueden contribuir a aclarar los probables efectos de los procesos sociales comunitarios en la delincuencia juvenil. En concreto, sobre la base de la literatura consultada se establecen relaciones indirectas entre la comunidad y la delincuencia mediante la conducta parental (apoyo y supervisión) y el contacto con amigos antisociales. Los signos que acompañan a los path indican la dirección de la 
relación de una variable independiente y otra dependiente, los cuales son congruentes con la contribución empírica y teórica en el área.

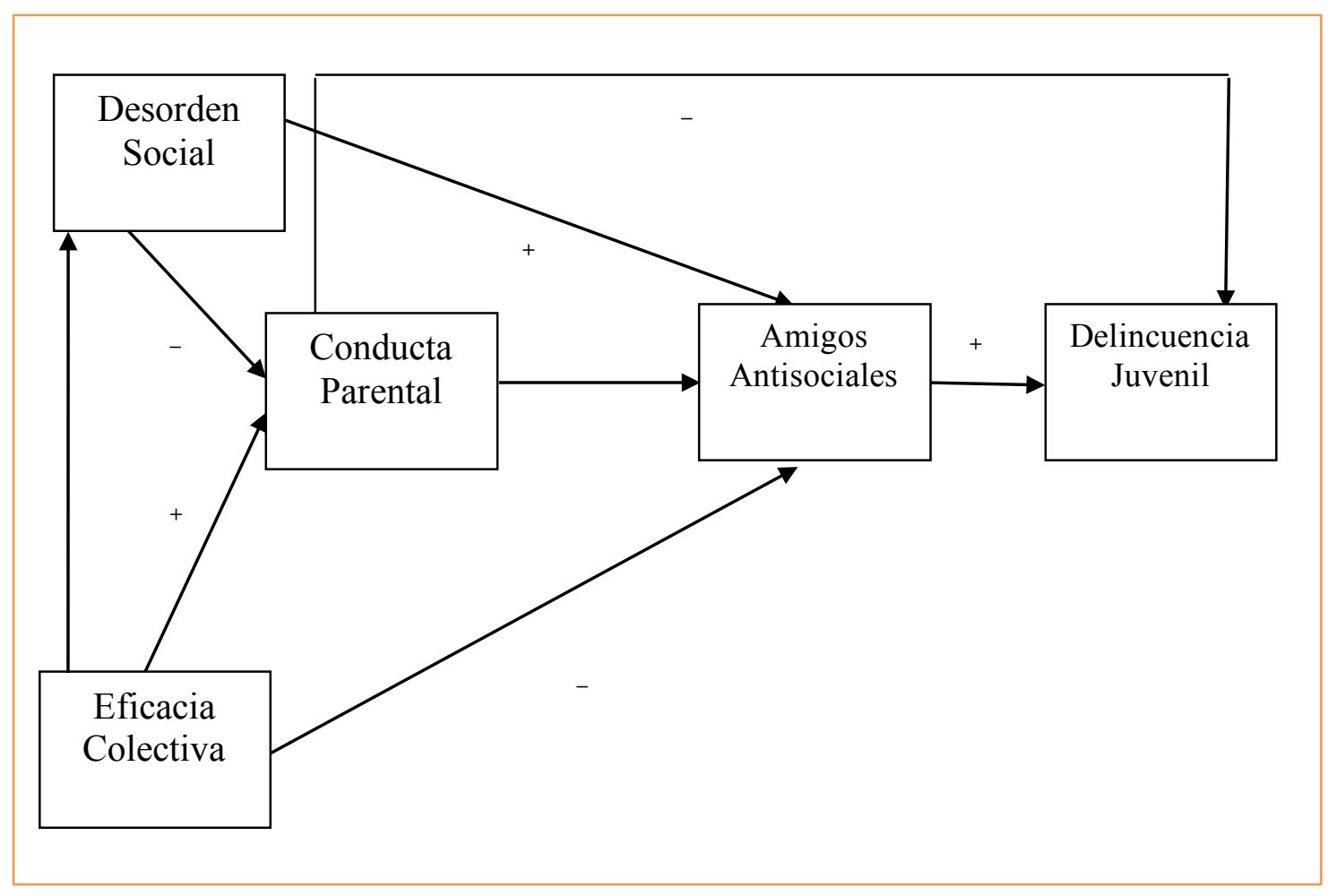

Figura 1. Modelo conceptual de la relación entre comunidad y delincuencia juvenil.

\section{Método}

\subsection{Procedimiento y características de la muestra}

Los datos se obtuvieron de un estudio transversal en el que participaron 1290 adolescentes de 11 a 19 años. Este estudio se llevó a cabo entre noviembre de 2012 y febrero de 2013. Formaron parte de esta investigación 8 liceos públicos de la ciudad de Mérida, Venezuela. Con carácter previo a la aplicación de los autoinformes, se gestionó el permiso de los directores de cada uno de los planteles participantes y, mediante estas mismas personas, se obtuvieron los consentimientos informados de los padres. Asimismo, la investigación se presentó como una actividad absolutamente voluntaria y los alumnos que no estuvieron de acuerdo con la misma se retiraron. Los cuestionarios 
de autoinforme fueron llenados en los planteles, en secciones de más o menos 30 estudiantes. Los profesores recibieron instrucciones por parte de los asistentes de investigación de cómo administrar el instrumento y, además, se garantizó el riguroso respeto de la confidencialidad y el anonimato. Incluso, antes, se les expuso a los directores de plantel la privacidad de la información suministrada por los participantes del estudio. Solo uno de los planteles escogidos se negó a tomar parte de la investigación. Del total de cuestionarios aplicados se excluyeron 279 (22\%) por problemas de llenado, inconsistencias en las respuestas, falta de información, etc. La muestra final estuvo constituida por 1011 sujetos.

El tipo de muestreo elegido para esta investigación fue no probabilístico. Por dificultades presupuestarias se resolvió usar una muestra por conveniencia. Los planteles educativos, que han colaborado en estudios anteriores, se localizan en comunidades en las que habitan familias con un nivel socioeconómico medio-bajo. En total, formaron parte de este estudio $470(46,9 \%)$ chicos y $532(53,1 \%)$ chicas. La edad promedio de estos adolescentes fue de 14, 46 años (D.T: 1,77). Un 53,2\% de la muestra reportó vivir con ambos padres, una cuarta parte con su madre y hermanos, 6,2\% solo con su madre y $1,4 \%$ solo con su padre. El 33\% de las madres de estos jóvenes son amas de casa, una cuarta parte profesionales, $26 \%$ trabajadoras calificadas y $15,1 \%$ no calificadas. Un $20 \%$ de los padres son profesionales, $45 \%$ trabajadores calificados y un tercio desempeñan trabajos no calificados.

\subsection{Variables}

\section{Conducta parental}

El Abusive and Supportive Environments Parenting Inventory (EASE-PI) de Nicholas y Bieber (1997), es un cuestionario elaborado para evaluar el apoyo y el maltrato que se da en el entorno familiar. En esta investigación se consideró la subescala de apoyo (12 ítems) para medir esta forma de conducta parental (ver lista de los ítems de esta escala en el Anexo). Aplicando una escala tipo Likert de 5 puntos que va de 0 (Nunca) a 4 (Con mucha frecuencia), los adolescentes reportaron la frecuencia con la que perciben ayuda, consuelo y afecto de sus padres. En la presente investigación, el alfa de Cronbach conseguido para el apoyo de la madre fue de .94 y para el padre de .96 . 
Para medir la supervisión familiar se adoptó el Parental Monitoring Index de Esbensen y Weerman (2005). Para esta investigación se ajustaron 3 de los ítems originales y se añadieron 2 más para evaluar la supervisión paterna con los amigos (ver lista de los ítems de esta escala en el Anexo). Las categorías de respuesta también se presentan en escala tipo Likert que varía de 0 (Nunca/A ninguno) a 3 (Siempre/A todos) puntos. Se obtuvo un coeficiente alpha de Cronbach de .70.

\section{Amigos antisociales}

Se decidió elaborar un grupo de ítems para estimar la conducta antisocial y delincuencia de los amigos. Estos indicadores, aun cuando son propios, se soportan en la revisión de la literatura en el área. Esta variable se midió preguntándole al adolescente sobre la cantidad de amigos residentes en su comunidad que incurren en consumo de drogas ilegales, vandalismo, agresión, robo y, además, que portan armas y han sido arrestados por la policía (ver lista de los ítems en el Anexo). El rango de respuesta, formulado en escala tipo Likert, varía entre 0 (Ninguno) y 3 (Todos) puntos. El coeficiente de confiabilidad alpha de Cronbach fue de .83 .

\section{Percepción de desorden social y eficacia colectiva}

Es importante acotar que para medir los efectos de la comunidad, en este estudio se asume un enfoque subjetivo fundamentado principalmente en información a nivel individual. Con referencia a esto, Gracia, Fuentes y García (2010) comentan que para estudiar la influencia de la comunidad en la conducta de los jóvenes hay cierto acuerdo en lo valioso de ofrecer mayor importancia a las experiencias y percepciones sobre las condiciones de este entorno. En algunos casos resulta más útil este tipo de información con respecto a las medidas objetivas como, por ejemplo, las basadas en censos o en registros de unidades geográficas pre-determinadas. Una razón es que este tipo de medidas no son totalmente coherentes con la manera como la comunidad es vista por sus residentes. Es más, para algunos autores son las percepciones de la comunidad las que afectan la conducta mediante su impacto en el adolescentes y en el funcionamiento familiar (Ver Roosa, White, Zeiders y Tein, 2009, para una revisión).

La percepción de desorden social se evaluó con algunos ítems adaptados de una medida empleada en la Encuesta Internacional de Autoinforme (ISRD-2) (Birkbeck, 
Morillo y Crespo, 2010). A los adolescentes se les consultó sobre 7 características relacionadas con la presencia en su zona residencial de violencia, delincuencia, comercio y consumo de alcohol/drogas y escándalo en espacios públicos (ver lista de los ítems en el Anexo). Los estudiantes respondieron a una escala Likert de 4 puntos que oscila entre 0 (Totalmente en desacuerdo) y 3 (Totalmente de acuerdo). La consistencia interna hallada fue de .88 (alpha Cronbach).

La percepción de eficacia colectiva fue estimada con tres ítems tomados también de la Encuesta Internacional de Autoinforme (ISRD-2) (Birkbeck et al., 2010) y que han sido empleados en otras investigaciones sobre contextos comunitarios (v. gr. Cohen, Inagami y Finch, 2008). El primero hace referencia al vínculo entre los vecinos (v. gr. Los vecinos está muy unidos en mi comunidad), el segundo, al hecho de si los vecinos son personas que generan confianza (v. gr. Los vecinos de mi comunidad son de confiar) y el tercero evalúa el control social informal (v. gr. Mis vecinos se dan cuenta cuando me estoy portando mal y me lo hacen saber) (ver lista de los ítems en el Anexo). Los participantes calificaron cada ítem en una escala Likert de 4 puntos. Para esta investigación se construyó un indicador de eficacia colectiva sumando las puntuaciones de los tres ítems. La confiabilidad fue de .70 (alpha Cronbach).

\section{Delincuencia juvenil}

En esta investigación se usó una adaptación del Cuestionario de Conductas Antisociales (CCA) propuesto originalmente por Luengo y su grupo de investigación (Luengo, Carrillo, Otero y Romero, 1994; Mirón y Otero-López, 2005). Con este auto-informe se midieron 21 ítems relacionados con delincuencia juvenil (ver lista de los ítems en el Anexo). Es conveniente aclarar que estas variables evalúan acciones que, de ser detectadas por los órganos de control social de este país, se convertirían en delito. Por ejemplo, se evaluó si el adolescente ha incurrido en algunos comportamientos como lesiones personales, robo, hurto, porte ilícito de arma, instigación para delinquir, intento de homicidio, desacato a la autoridad y tráfico de drogas. Las categorías de respuesta presentadas en formato tipo Likert varían de 0 (Nunca) a 4 (Siempre) puntos. Para calcular un índice global de delincuencia juvenil se sumaron las puntuaciones de los 21 ítems. El alfa de Cronbach obtenido fue de 89. 


\section{Resultados}

\subsection{Descriptivos y coeficientes de correlación de Pearson}

La Tabla 1 resume los estadísticos descriptivos de las variables de estudio. En promedio, los adolescentes de esta muestra perciben altos niveles de apoyo tanto de la madre como del padre, así como de supervisión parental. El número de amigos que, por término medio, están implicados en conductas antisociales es muy bajo; pero, al examinar el rango observado, se encuentran jóvenes que reportan que la mayoría de sus amigos han incurrido en casi todas las conductas antisociales evaluadas.

Con base en estos resultados, los niveles de desorden social percibido son, en promedio, de moderados a bajos; aunque, el rango observado revela que hay sujetos que perciben muy altos niveles de incivilidad en sus zonas residenciales. Por término medio, los niveles de eficacia colectiva percibida también son de moderados a bajos aun cuando la variabilidad de las puntuaciones en este indicador es considerable, toda vez que hay adolescentes que revelan no percibir eficacia colectiva en su comunidad y, al contrario, otros que perciben altos niveles. Sin embargo, no se observa ningún adolescente que consiga puntuar el nivel máximo de eficacia colectiva percibida medido en este estudio. Por último, se puede detallar que la máxima puntuación conseguida en la escala de delincuencia juvenil fue de 44 puntos. La incidencia de conductas que pudieran ser susceptibles de sanción jurídica es, en promedio, muy baja en esta muestra $^{2}$

Esta tabla también contiene el patrón de relaciones entre las variables de estudio. Hay que destacar el hecho de que todas las correlaciones fueron significativas y se dieron en la dirección esperada. En particular, se observa que el apoyo de la madre y del padre, así como su supervisión, se relacionan negativamente con la delincuencia de los hijos. Interactuar con amigos que experimentan comportamientos antisociales se asocia positivamente con la actividad delictiva de los adolescentes. Desde una perspectiva contextual, el desorden social percibido correlaciona positivamente con la desviación

\footnotetext{
2 Como dato adicional, el $70 \%$ de los adolescentes de esta muestra reporta nunca haber experimentado conductas delictivas, con respecto a un $30 \%$ que sí ha incurrido en alguna de ellas, lo que significa que también la prevalencia de este tipo de comportamientos es baja.
} 
juvenil mientras que la percepción de eficacia colectiva, al contrario, lo hace de forma negativa. En este caso, las variables que tienen una relación más fuerte con la variable criterio son los amigos antisociales $(r=.52, p \leq .001)$ y el desorden social percibido $(r=$ $.25, p \leq .001)$.

Tabla 1. Rangos, medias, desviación típica y correlaciones entre las variables observadas en el análisis $(\mathrm{N}=1011)$

\begin{tabular}{|l|l|l|l|l|l|l|l|l|l|l|}
\hline & R.T. & R.O. & $\boldsymbol{M}$ & $\boldsymbol{D T}$ & $\mathbf{1}$ & $\mathbf{2}$ & $\mathbf{3}$ & $\mathbf{4}$ & $\mathbf{5}$ & $\mathbf{6}$ \\
\hline 1. Apoyo Madre & $0-48$ & $0-48$ & 37,7 & 11,0 & - & & & & & \\
\hline 2. Apoyo Padre & $0-48$ & $0-48$ & 32,1 & 14,4 & $.55^{* * *}$ & - & & & & \\
\hline 3. Supervisión Parental & $0-15$ & $1-15$ & 10,2 & 2,9 & $.37^{* * *}$ & $.33^{* * *}$ & - & & & \\
\hline 4. Amigos Antisociales & $0-21$ & $0-20$ & 2,0 & 2,9 & $-.17^{* * *}$ & $-.14^{* * *}$ & $-.24^{* * *}$ & - & & \\
\hline 5. Desorden Social & $0-21$ & $0-21$ & 8,0 & 5,8 & $-.10^{* *}$ & $-.10^{* *}$ & $-.10^{* *}$ & $.33^{* * *}$ & - & \\
\hline 6. Eficacia Colectiva & $0-9$ & $0-8$ & 4,3 & 1,9 & $.18^{* * *}$ & $.17^{* * *}$ & $.13^{* * *}$ & $-.16^{* * *}$ & $.34^{* * *}$ & - \\
\hline 7. Delincuencia Juvenil & $0-84$ & $0-44$ & 1,6 & 4,7 & $-.18^{* * *}$ & $-.11^{* *}$ & $-.20^{* * *}$ & $.52^{* * *}$ & $.25^{* * *}$ & $-.11^{* *}$ \\
\hline
\end{tabular}

$* p \leq .05 ; * * p \leq .01 ; * * * p \leq .001$; Nota: R.T: Rango Teórico, R.O: Rango Observado.

Hay que destacar, además, que las variables apoyo y supervisión parental se asocian de manera negativa con amigos antisociales y desorden social percibido. En contraste, estas mismas características familiares correlacionan positivamente con la percepción de eficacia colectiva. La relación entre el grupo de amigos antisociales y el desorden social percibido es de signo positivo ( $r=.33, p \leq .001)$; y, en cambio, el de la asociación con la eficacia colectiva percibida es negativo $(r=-.16, p \leq .001)$. Por último, se observa una correlación negativa entre la desorganización social representada por la incivilidad y la percepción de eficacia colectiva $(r=-.34, p \leq .001)$.

\subsection{Análisis de Estructuras de Covarianzas}

Inicialmente se ha puesto a prueba un modelo completo o saturado que incluye todas las relaciones posibles de acuerdo al modelo teórico asumido. El propósito de esta estrategia es obtener un modelo más parsimonioso tal cual lo hicieron Chung y Steinberg (2006), Mrug y Windle (2009) y Vieno et al., (2010) y contrastar a partir de ahí las hipótesis de 
partida. El ajuste del modelo se evaluó empleando el programa AMOS 17. Es preciso apuntar que el coeficiente de Mardia obtenido en este estudio fue de 59,8. Este coeficiente es inferior a 70, lo que significa que no hay un problema substancial con respecto a la distribución normal multivariada. En todo caso, el método de estimación de parámetros escogido fue el de Máxima Verosimilitud porque es suficientemente robusto y aporta estimaciones estables, eficientes y no sesgadas ante la falta de normalidad.

Tal como se observa en la Tabla 2, los índices de bondad de ajuste entre el modelo saturado y los datos empíricos de esta muestra no son por completo adecuados ${ }^{3}$. En concreto, el valor de CMIN/DF excede el umbral de 3, el $\mathrm{P}$ ratio indica un bajo nivel de parsimonia y el RMSEA presenta el valor límite convenido de .08. Los coeficientes path estandarizados del modelo saturado se presentan en la Tabla 3.

Tabla 2. Índices de bondad de ajuste del modelo saturado

\begin{tabular}{|l|l|l|l|l|l|l|l|l|l|l|}
\hline & $\mathbf{X}^{\mathbf{2}}$ & g.l. & $\boldsymbol{p}$ & $\begin{array}{c}\text { CMIN/ } \\
\text { DF }\end{array}$ & NFI & CFI & GFI & P ratio & TLI & RMSEA \\
\hline & & & & & & & & & & \\
\hline Modelo saturado & 32,3 & 8 & .000 & 4,04 & .95 & .96 & .98 & .38 & .89 & .08 \\
\hline
\end{tabular}

Ahora bien, con la finalidad de reducir y lograr un modelo más parsimonioso, se decidió evaluar con mayor detalle la significatividad de los parámetros (relaciones entre variables) y se decidió mantener en el modelo sólo aquellas relaciones que resultaron estadísticamente significativas después de varias iteraciones. En consecuencia, se eliminó del modelo saturado (ver Figura 2) la relación entre desorden social percibido y la variable latente conducta parental, la ruta entre eficacia colectiva percibida y amigos antisociales, la relación directa de eficacia colectiva percibida y delincuencia juvenil y el efecto directo entre conducta parental y delincuencia juvenil. Estas relaciones se rechazaron porque el nivel de significación excedió el umbral de .05. Además, es importante destacar que de acuerdo con Mrug y Windle (2009) es recomendable eliminar en este proceso de evaluación las relaciones significativas que no están establecidas en el

\footnotetext{
${ }^{3}$ Para $\mathrm{Hu}$ y Bentler (1995) un modelo presenta un buen ajuste a los datos empíricos cuando el producto entre el Chi-cuadrado y los grados de libertad (CMIN/DF) es menor a 3, los índices de ajuste NFI, CFI, GFI y TLI son iguales o superiores a 0.90 y el RMSEA es menor a 0.08 . Además, se considera que un modelo presenta más parsimonia cuando los valores del índice $\mathrm{P}$ ratio son mayores.
} 
modelo hipotético de partida. Por consiguiente, se decidió suprimir la relación directa del desorden social percibido y la delincuencia juvenil.

Tabla 3. Coeficientes path estandarizados para el modelo saturado

\begin{tabular}{|l|l|l|l|l|l|}
\hline & $\begin{array}{c}\text { Desorden } \\
\text { Social }\end{array}$ & $\begin{array}{c}\text { Eficacia } \\
\text { Colectiva }\end{array}$ & $\begin{array}{c}\text { Conducta } \\
\text { Parental }\end{array}$ & $\begin{array}{c}\text { Amigos } \\
\text { Antisociales }\end{array}$ & $\begin{array}{c}\text { Delincuencia } \\
\text { Juvenil }\end{array}$ \\
\hline 1. Desorden Social & - & $-24 * * *$ & -07 & $.27 * * *$ & $.11^{* *}$ \\
\hline 2. Eficacia Colectiva & & - & $.26 * * *$ & .02 & .05 \\
\hline 3. Conducta Parental & & & - & $-.29 * * *$ & $-.10^{*}$ \\
\hline 3. Amigos Antisociales & & & & - & $.45^{* * *}$ \\
\hline
\end{tabular}
${ }^{*} p \leq .05 ; * * p \leq .01 ; * * * \leq .001$.

De este modo, se obtuvo un modelo reducido más parsimonioso cuyos índices de ajuste se presentan en la Tabla 4. Como puede observarse, los diferentes índices valorados (siguiendo las propuestas de $\mathrm{Hu}$ y Bentler, 1995) muestran un mejor ajuste de este modelo con respecto al ajuste del modelo saturado, en especial, el producto CMIN/DF, $\mathrm{P}$ ratio, TLI y RMSEA. En concreto, el ajuste del modelo final se logró por eliminar las relaciones no significativas referidas en el párrafo anterior y el efecto directo del desorden social percibido en la delincuencia juvenil. La diferencia entre los valores del $\mathrm{X}^{2} \mathrm{y}$ los grados de libertad entre el modelo saturado y el reducido es de $\Delta \mathrm{X}^{2}(5)=10,2, n s$.

Tabla 4. Índices de bondad de ajuste del modelo reducido

\begin{tabular}{|l|c|c|c|c|c|c|c|c|c|c|}
\hline & \multicolumn{1}{|c|}{$\mathbf{X}^{2}$} & g.l. & $\boldsymbol{p}$ & $\begin{array}{c}\text { CMIN/ } \\
\text { DF }\end{array}$ & NFI & CFI & GFI & P ratio & TLI & RMSEA \\
\hline $\begin{array}{l}\text { Modelo } \\
\text { reducido }\end{array}$ & 42,5 & 13 & .000 & 3,26 & .93 & .95 & .98 & .62 & .91 & .06 \\
\hline
\end{tabular}

Así, el modelo reducido queda configurado de acuerdo con los datos presentados en la Figura 2. Se observa, en primer lugar, que los indicadores escogidos para formar la variable latente conducta parental son apropiados. El apoyo de la madre y del padre presentan cargas factoriales que exceden el .60 y la supervisión un peso equivalente a .50.

En segundo lugar, en cuanto a los efectos directos, la percepción de eficacia colectiva presenta un efecto negativo en el desorden social percibido $(\gamma=-.24, \mathrm{p} \leq .001)$; $\mathrm{y}$, en contraste, una relación directa con la conducta parental basada en el apoyo y la supervisión $(\gamma=.27, \mathrm{p} \leq .001)$. Por su parte, el desorden social percibido tiene un efecto 
positivo en los amigos antisociales $(\beta=.27, \mathrm{p} \leq .001)$. La conducta parental, en cambio, presenta una relación negativa con amigos antisociales $(\beta=-.28, \mathrm{p} \leq .001)$. La variable amigos antisociales tiene un efecto directo en la delincuencia juvenil $(\beta=.50, p \leq .001)$. Con respecto a esto, los amigos antisociales constituyen el elemento más relevante en cuanto al desarrollo de conductas delictivas en este modelo.

En tercer lugar, cabe señalar (ver Tabla 5) los efectos indirectos siguientes: la percepción de eficacia colectiva presenta un efecto indirecto en la delincuencia juvenil mediante la conexión con el desorden social percibido, la conducta parental y los amigos antisociales $(\beta=-.07, \mathrm{p} \leq .05)$. Esta misma característica del vecindario incide indirectamente en tener amigos antisociales por medio de la conducta parental y del desorden social percibido $(\beta=-.14, \mathrm{p} \leq .001)$. La percepción de desorden social en la comunidad tiene un efecto indirecto en la delincuencia del adolescente mediante los amigos antisociales $(\beta=.14, \mathrm{p} \leq .001)$. Y, sumado a esto, la familia se relaciona indirectamente con la delincuencia juvenil por intermedio de los amigos antisociales $(\beta=$ $-.14, \mathrm{p} \leq .001)$.

Por último, la explicación conjunta que las variables del modelo proporcionan sobre la delincuencia juvenil es moderada $\left(\mathrm{R}^{2}=.25\right)$. 


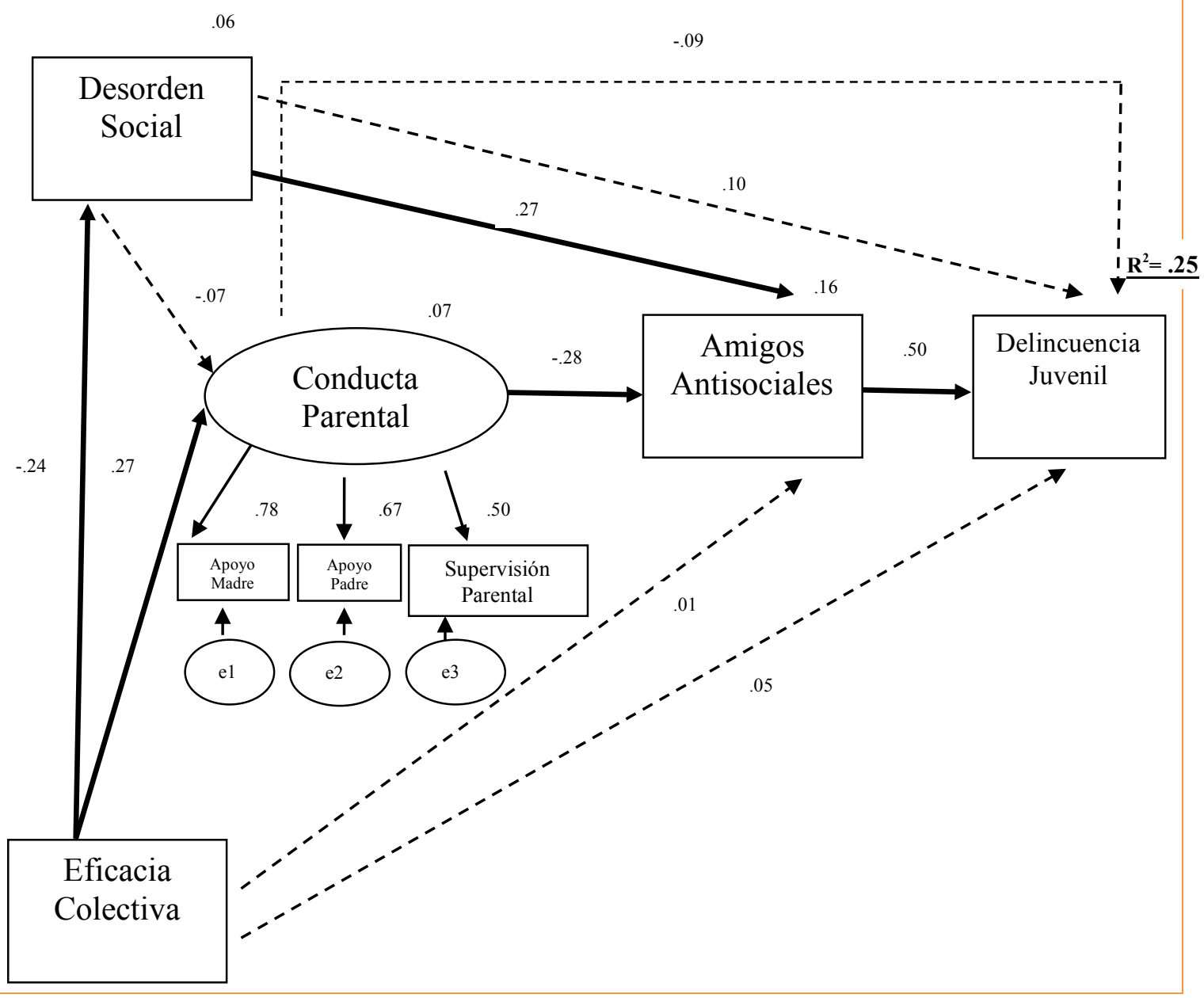

Nota: Las relaciones entre variables representadas por una línea continua fueron significativas a un nivel de $p=\leq .001$. Las relaciones entre variables representadas por una línea interrumpida no resultaron significativas, excepto la relación entre desorden social percibido y delincuencia juvenil $(p \leq .05)$ que fue eliminada en el proceso de ajuste por no ser una de las hipótesis del modelo.

Figura 2. Modelo mediacional final de la relación comunidad y delincuencia juvenil $(\mathrm{N}=537)$. 
Tabla 5. Efectos totales, directos e indirectos estandarizados del Modelo reducido

\begin{tabular}{|c|c|c|c|c|c|c|c|c|c|c|c|c|}
\hline & \multicolumn{4}{|c|}{ Efectos totales } & \multicolumn{4}{|c|}{ Efectos directos } & \multicolumn{4}{|c|}{ Efectos indirectos } \\
\hline & $\begin{array}{c}\text { Eficacia } \\
\text { Colectiva }\end{array}$ & $\begin{array}{c}\text { Desorden } \\
\text { Social }\end{array}$ & $\begin{array}{l}\text { Conducta } \\
\text { Parental }\end{array}$ & $\begin{array}{c}\text { Amigos } \\
\text { Antisociales }\end{array}$ & $\begin{array}{l}\text { Eficacia } \\
\text { Colectiva }\end{array}$ & $\begin{array}{c}\text { Desorden } \\
\text { Social }\end{array}$ & $\begin{array}{c}\text { Conducta } \\
\text { Parental }\end{array}$ & $\begin{array}{c}\text { Amigos } \\
\text { Antisociales }\end{array}$ & $\begin{array}{l}\text { Eficacia } \\
\text { Colectiva }\end{array}$ & $\begin{array}{c}\text { Desorden } \\
\text { Social }\end{array}$ & $\begin{array}{l}\text { Conducta } \\
\text { Parental }\end{array}$ & $\begin{array}{c}\text { Amigos } \\
\text { Antisociales }\end{array}$ \\
\hline 1. Desorden Social & -.24 & .00 & .00 & .00 & -.24 & .00 & .00 & .00 & .00 & .00 & .00 & .00 \\
\hline 2. Conducta Parental & .27 & .00 & .00 & .00 & .27 & .00 & .00 & .00 & .00 & .00 & .00 & .00 \\
\hline 3. Amigos Antisociales & -.14 & .27 & -.28 & .00 & .00 & .27 & -.28 & .00 & -.14 & .00 & .00 & .00 \\
\hline 4. Apoyo de la madre & .21 & .00 & .78 & .00 & .00 & .00 & .78 & .00 & .21 & .00 & .00 & .00 \\
\hline 5. Apoyo del padre & .18 & .00 & .67 & .00 & .00 & .00 & .67 & .00 & .18 & .00 & .00 & .00 \\
\hline 6. Supervisión familiar & .14 & .00 & .50 & .00 & .00 & .00 & .50 & .00 & .14 & .00 & .00 & .00 \\
\hline 7. Delincuencia Juvenil & -.07 & .14 & -.14 & .50 & .00 & -00 & .00 & 50 & -.07 & .14 & -.14 & .00 \\
\hline
\end{tabular}




\section{Discusión y conclusiones}

\subsection{Discusión}

El objetivo principal de esta investigación ha sido evaluar un modelo de mediación sobre las relaciones entre la percepción de eficacia colectiva y desorden social, conducta parental, amigos desviados y delincuencia juvenil en una muestra de adolescentes escolarizados de Venezuela. Con base en el modelo integrador puesto a prueba, se han corroborado algunas relaciones que son consistentes con los hallazgos de investigaciones previas.

Para empezar, en lo que concierne a la comunidad estos resultados indican que la percepción de eficacia colectiva es una variable asociada negativamente con el desorden social percibido en el vecindario. Problemas sociales como pelear en la calle, consumir alcohol, traficar con drogas y escuchar música a alto volumen parecen ser inhibidos si los residentes presentan cohesión en términos de confianza y, además, expectativas comunes para la acción (Sampson, 2004). Con independencia de algunas diferencias metodológicas, estos resultados parecen ser coherentes con los reportados por Sampson y Raudenbush (2001) para comunidades pobres y violentas de EE.UU y, en general, con las predicciones de Sampson y su equipo de investigación (1997). Asimismo, los datos corroboran que la eficacia colectiva percibida tiene una asociación positiva con la conducta parental. Hallazgos similares han sido reportados por Rankin y Quane (2002), Chung y Steinberg (2006), Vieno et al. (2010) y considerados teóricamente por Wikström y Sampson (2003). Aun cuando Rankin y Quane hallan solo un efecto de la eficacia colectiva en el monitoreo, y no en el apoyo de los padres, demuestran que las zonas residenciales con mayores niveles de eficacia colectiva presentan también mayores niveles de supervisión parental. Quizás el clima normativo de las comunidades que 'funcionan bien' y son eficaces, estimula a los padres a supervisar mejor a sus hijos. Simultáneamente, según el modelo puesto a prueba el desorden social percibido se asocia al contacto con entornos de amigos desviados. Estos resultados son congruentes con los conseguidos por Shaw y McKay (1942), Sampson y Groves (1989) y Chung y Steinberg (2006) pero contrarios a los reportados por Mrug y 
Windle (2009). En esta dirección, es plausible que las comunidades que presentan problemas de incivilidad y poco control social informal incrementen la oportunidad de sostener vínculos de amistad con otros jóvenes que experimentan conductas contrarias a la norma.

De la misma forma, los resultados de este estudio apuntan a que la conducta parental eficaz se relaciona negativamente con la participación de los hijos en grupos de iguales problemáticos. Este hallazgo es coherente con un número importante de investigaciones anteriores que han relacionado el tipo de conducta parental desarrollada en el hogar con la influencia de los amigos desviados (Dishion, Poulin y Burraston, 2001; Jessor y Jessor, 1977; Mirón y Otero-López, 2005; Rankin y Quane, 2002; Rodríguez, 2011; Scaramella et al., 2002). Finalmente, el modelo reducido también confirma la asociación entre tener amigos desviados y la propia delincuencia del adolescente. La observación sistemática ha evidenciado recurrentemente que la participación en grupos de amigos antisociales es la influencia más próxima a la toma de contacto con la desviación durante la adolescencia (Rodríguez et al., 2012; Rodríguez, 2011; 2010; Rodríguez y Mirón, 2008; Mirón y Otero-López, 2005).

Por otra parte, en el modelo reducido se comprueban algunos efectos indirectos de la percepción de desorden social y eficacia colectiva en la conducta delictiva. El primero de ellos indica que la relación negativa entre la percepción de eficacia colectiva y la delincuencia juvenil se explica por el desorden social percibido, la familia y el grupo de amigos. Aun cuando la fuerza y el nivel de significación de esta relación no son tan robustos $(\beta=-.07, \mathrm{p} \leq .05)$, los resultados confirman que las comunidades con altos niveles de eficacia tienen la capacidad de disminuir los actos de incivismo y de aumentar la conducta parental eficaz. Tal vez al haber presencia de bajos niveles de desorden social y tácticas de gestión parental efectivas que se sustentan en el afecto y la supervisión, la posibilidad de interactuar con amigos antisociales es baja. Por consiguiente, hay poca probabilidad de que los chicos incurran en actos delictivos. Estos resultados son coherentes con los reportados por Rankin y Quane (2002) y Chung y Steinberg (2006).

El segundo efecto indirecto indica que el desorden social percibido se relaciona con la delincuencia por medio del grupo de amigos desviados. Como se comentó en el párrafo anterior, esto significa que aquellas zonas residenciales con problemas de 
incivilidad (exposición a factores como la violencia, venta y consumo de alcohol/drogas y escándalos públicos) constituyen espacios favorables para la interacción con grupos de adolescentes desviados y la toma de contacto con la delincuencia. La inferencia principal es que la desorganización social, en este caso el desorden social, influye en las formas de socialización informal de los adolescentes $\mathrm{y}$, en consecuencia, en su comportamiento. La premisa de que la influencia de la comunidad es probablemente indirecta y actúa mediante contextos de socialización más próximos coincide con los hallazgos de Rankin y Quane (2002), Tolan et. al., (2003), Chung y Steinberg (2006) y Byrnes et al. (2011) y también con los encontrados en la presente investigación. En tal sentido, los resultados de este estudio representan un apoyo parcial a la hipótesis de los efectos indirectos de la comunidad en la delincuencia juvenil por intermedio de los agentes de socialización primaria.

Del mismo modo, también es fundamental considerar algunas hipótesis que no fueron confirmadas por los datos. Así, a diferencia de la percepción de eficacia colectiva, la relación positiva del desorden social percibido y la actividad delictiva no está mediatizada por las prácticas parentales. La influencia de este proceso comunitario se transmite exclusivamente por medio de los amigos antisociales (e incluso, quizá también de forma directa como permiten apreciar algunos análisis parciales), y no mediante el funcionamiento familiar basado en el apoyo y la supervisión. La ausencia de tales efectos indirectos discrepa de los resultados de otros estudios (v. gr. Chung y Steinberg, 2006; Mrug y Windle, 2009) que han señalado la actuación de la familia en la asociación entre comunidad y delincuencia juvenil. Pero ¿por qué la conducta parental no es afectada por el desorden social percibido y, por ende, no interviene en la relación de esta característica comunitaria y la delincuencia juvenil? Una respuesta de algún modo especulativa, desprovista de soporte empírico, puede ser la siguiente: dadas las especificidades culturales de la ciudad de Mérida y, en general, de Venezuela con respecto a los altos niveles de inseguridad y delincuencia, la cotidianidad de este fenómeno ha generado en los ciudadanos una suerte de habituación al mismo. Este posible proceso de adaptación en el caso de los padres, no permite que la exposición a ciertas señales problemáticas de desorganización comunitaria (v. gr. delincuencia, tráfico de drogas, peleas, consumo de alcohol y drogas, etc.) constituya un factor estresante que actúe negativamente en la calidad de la crianza. No obstante, la ausencia 
de esta relación y, por consiguiente, los contrastes con otros estudios (v. gr. Byrnes et al., 2011; Leventhal y Brooks-Gunn, 2000; Simons et al., 1996; Stern y Smith, 1995) puede deberse también a un artificio metodológico que involucra tanto a las medidas y constructos como a la fuente de información elegida en esta investigación.

\subsection{Limitaciones}

Además, resulta oportuno adelantar que los efectos indirectos aquí contrastados deben interpretarse con cierta precaución y, en consecuencia, es oportuno reflexionar sobre algunas limitaciones de este estudio.

En primer lugar, la principal limitación es el carácter transversal de los datos. Dada la naturaleza no longitudinal de los mismos, la dirección de las relaciones directas e indirectas no debe interpretarse de manera causal.

En segundo lugar, otra limitación tiene que ver con la medición. Por ejemplo, para medir las características del desorden social y la eficacia colectiva se han utilizado exclusivamente indicadores subjetivos que pueden alterar las relaciones encontradas entre las variables. Para contrarrestar esto, Sampson y Raudenbush (2001) propugnan el uso de métodos distintos al auto-informe como manera de obtener información 'neutra y objetiva' de las comunidades. En particular, han hecho mediciones mediante una técnica que denominan 'observación social sistémica'.

En tercer lugar, en este modelo hipotético de relaciones no se incorporaron factores estructurales. Resultaría valioso incluir en otros modelos conceptuales variables como el nivel socioeconómico, índices de viviendas alquiladas u otros indicadores socio-demográficos, porque la literatura sostiene que afectan directamente a los diferentes procesos sociales que se dan en una comunidad (Gracia y Herrero, 2006) e indirectamente a la delincuencia juvenil. Por último, en este estudio no hay una gran diversidad en la muestra en cuanto a nivel socioeconómico y tampoco a tipo de comunidad en la que viven los jóvenes encuestados.

\subsection{Conclusión e implicaciones}


Los resultados de este estudio apuntan a que las zonas residenciales de algunos jóvenes pueden estar caracterizadas por problemas de organización y coexistencia. En ambientes así, los patrones delictivos de aquellos adolescentes que no experimentan una adecuada gestión parental pueden ser forjados y exacerbados por el contacto con grupos de amigos desviados. Tal vez los hallazgos obtenidos aquí puedan dar respuesta de manera provisional a la pregunta principal de este trabajo $\mathrm{y}$, también, ser evidencia que contribuya a la formulación de nuevas hipótesis desde un enfoque comunitario sobre la etiología de la conducta delictiva en nuestro país.

Para finalizar, en términos de gestión pública, este estudio deja claro que los esfuerzos deben ir dirigidos principalmente a promover comunidades de vecinos organizadas que gestionen su convivencia y, además, que trabajen en función de los problemas y propósitos colectivos.

\section{Agradecimientos}

Un agradecimiento especial al Consejo de Desarrollo, Científico, Humanístico y Tecnológico (CDCHT) de la Universidad de Los Andes. Mérida-Venezuela por el apoyo recibido. Igualmente, se agradece a los revisores de la REIC por sus importantes observaciones y contribuciones a este artículo.

\section{Financiación}

Este artículo forma parte del Proyecto de Investigación "Comunidad, contextos de socialización, autocontrol y delincuencia juvenil". Código D-443-12-09-B, financiado por el Consejo de Desarrollo, Científico, Humanístico y Tecnológico (CDCHT) de la Universidad de Los Andes. Mérida-Venezuela. 


\section{Referencias}

Birkbeck, C; Morillo, S. y Crespo, F. (2010). 'Venezuela'. En: Juvenile Delinquency in Europe and Beyond. Results of the Second International Delinquency Self-Report Study. Springer, New York, pp. 385-398.

Byrnes, H; Miller, B; Chen, M. y Grube, J. (2011). The roles of mothers' neighborhood perceptions and specific monitoring strategies in youths' problem behavior. Journal of Youth and Adolescence, 40, 347-360.

Chung, H. y Steinberg, L. (2006). Relations Between Neighborhood Factors, Parenting Behavior, Peer Deviance, and Delinquency Among Serious Juvenile Offenders. Developmental Psychology, 42 (2), 319-331.

Cohen, D.A; Inagami, S. y Finch, B. (2008). The built environment and collective efficacy. Health and Place, 14 (2), 198-208.

de Kemp, R; Scholte, R; Overbeek, G. y Engels, R. (2006). Early delinquency: The role of home and best friends. Criminal Justice and Behavior, 33, 488-510.

Deutsch, A; Crockett, L; Wolff, J. y Russell, S. (2012). Parent and peer pathways to adolescent delinquency: Variations by ethnicity and neighborhood context. Journal of Youth and Adolescence, 41, 1078-1094.

Dishion, T.J; Bulluck, B; y Granic, I. (2002). Pragmatism in modeling peer influence: Dynamics, outcomes, and change processes. Development and Psychopathology, 14, 969-981.

Dishion, T.J; Poulin, Fr. y Burraston, B. (2001). Peer Group Dynamics Associated with Iatrogenic Effects in Group Interventions Ruth High-Risk Young Adolescents. New Directions for Child and Adolescent Development, 91, 79-92.

Downes, D. y Rock, P. (2007). Sociología de la desviación. Barcelona: Gedisa.

Esbensen, F.A. y Weerman, F.M. (2005). Youth gangs and troublesome youth groups in the United States and the Netherlands. A cross-national comparison. European Journal of Criminology, 2 (1), 5-37.

Gottfredson, M. y Hirschi, T. (1990). A general Theory of Crime. Stanford: Stanford University.

Gracia, E; Fuentes, M. y García, F. (2010). Barrios de riesgo, estilos de socialización parental y problemas de conducta en adolescentes. Intervención Psicosocial, 19 (3), 265-278. 
Gracia, E. y Herrero, J. (2006). La comunidad como fuente de apoyo social: Evaluación e implicaciones en los ámbitos individual y comunitario. Revista Latinoamericana de Psicología, 38 (2), 327-342.

Gorman-Smith, D; Tolan, P. y Henry, DB. (2000). A developmental-ecological model of the relation of family functioning to patterns of delinquency. Journal of Quantitative Criminology, 16, 169-198.

Haynie, D; Silver, E; y Teasdale, B. (2006). Neighborhood characteristics, peer networks and adolescent violence. Journal Quantitative Criminology, 22, 147-169.

Hoeve, M; Dubas, J.S; Eischelsheim, V.I; van der Laan, P; Smeenk, W. y Gerris, J. (2009). The relationship between parenting and delinquency: A meta-analysis. Journal of Abnormal Child Behavior, 37, 749-775.

Hu, L.T. y Bentler, P.M. (1995). Evaluating model fit. En: R.H. Hoyle (eds.), Structural Equation Modeling. Thousand Oaks, California: Sage.

Jessor, R. y Jessor, S.L. (1977). Problem behavior and psychosocial development: A longitudinal study of youth. N.Y: Academic Press.

Lenzi, M; Vieno, A; Perkins, D; Pastore, M; Santinello, S. y Mazzardis, S. (2011). Perceived neighborhood social resources as determinants of prosocial behavior in early adolescence. American Journal of community psychology, 50 (1-2), 37-49.

Leventhal, T. y Brooks-Gunn, J. (2000). The neighborhoods they live in: The effects of neighborhood residence on child and adolescent outcomes. Psychological Bulletin, 126, 309-337.

Luengo, M.A; Carrillo, M.T; Otero, J.M. y Romero, E. (1994). A short-term longitudinal study of impulsivity and antisocial behaviour. Journal of Personality an Social Psychology, 66, 542-548.

Mirón, L. y Otero-López, J.M. (2005). Jóvenes delincuentes. España: Ariel, S. A.

Mrug, S. y Windle, M. (2009). Mediators of neighborhood influences on externalizing behavior in preadolescent children. Journal of Abnormal Child Psychology, 37, 265280 .

Nicholas, K. y Bieber, S. (1997). Assessment of perceived parenting behaviors: The Exposure to Abusive and Supportive Environments Parenting Inventory (EASE-PI). Journal of Family Violence, 12 (3), 275-291.

Patterson, G; Dishion, T. y Yoerger, K. (2000). Adolescent growth in new forms of problem behavior: Macro- and micro-peer dynamics. Prevention Science, 1, 3-13. 
Pretty, G.M. (2002). Young people's development of the community-minded self: Considering community identity, community attachment and sense of community. En A.T. Fisher, C. Sonn y B. Bishop (Eds.), Psychological sense of community: Research, applications, and implications (pp. 183-203). N.Y: Kluwer Academic/Plenum Publishers.

Rankin, B.H. y Quane, J.M. (2002). Social contexts and urban adolescent outcomes: The interrelated effects of neighborhoods, families, and peer on African-American youth. Social Problems, 49, 79-100.

Rodríguez, J.A. (2010). Bajo autocontrol y conducta antisocial. Revista CENIPEC, 29, 213-240.

Rodríguez, J.A. (2011). Conducta antisocial en grupo: Una aproximación mediante ecuaciones estructurales. Revista CENIPEC, 30, 237-264.

Rodríguez, J.A. y Mirón, L. (2008). Grupos de amigos y conducta antisocial. Capítulo Criminológico, 36 (4), 121-149.

Rodríguez, J.A; Mirón, L y Rial, J.A. (2012). Análisis de la relación entre grupos de iguales, vinculación familiar y escolar, autocontrol y conducta antisocial, en una muestra de adolescentes venezolanos. Revista de Psicología Social, 27 (1), 25-38.

Roosa, M. White, R. Zeiders, K. y Tein, J. (2009). An examination of the role of perceptions in neighborhood research. Journal of Community Psychology, 37, 327341.

Sampson, R. (2004). Neighborhood and community. Collective efficacy and community safety. New Economy, 11, 106-113.

Sampson, R. y Groves, B. (1989). Community structure and crime: Testing socialdisorganization Theory. American Journal of Sociology, 94 (4), 774-802.

Sampson, R. y Raudenbush, S. (2001). Disorder in Urban Neighbourhoods- Does it lead to crime? National Institute of Justice. Research in Brief, 1-6.

Sampson, R; Raudenbush, S. y Earls, F. (1997). Neighborhoods and violent crime: A multilevel study of collective efficacy. Science, 277, 918-924.

Scaramella, LV; Conger, RD; Spoth, R. y Simons, R.L. (2002). Evaluation of a social contextual model of delinquency: A cross-study replication. Child Development, 73, 175-195.

Simons, RL; Johnson, C; Beaman, J; Conger, R y Whitbeck, L (1996). Parents and peer group as mediator of the effect of neighborhood on adolescent problem behavior. American Journal of Community Psychology, 24 (1), 145-171. 
Shaw, C. y McKay, H. (1942). Juvenile delinquency and urban areas. Chicago: University of Chicago.

Stern, S. y Smith, C. (1995). Family processes and delinquency in an ecological context. Social Service Review, 69, 703-731.

Tolan, P.H; Gorman-Smith. D. y Henry, D. (2003). The developmental ecology of urban males' youth violence. Developmental Psychology, 39, 274-291.

Vieno, A; Nation, M; Perkins, D; Pastore, M. y Santinello, M. (2010). Social capital, safety, concerns, parenting, and early adolescents' antisocial behavior. Journal of community psychology, 38 (3), 314-328.

Wilson, J. y Kelling, G. (1982). The Police and Neighborhood Safety: Broken Windows. Atlantic Monthly, 249 (3), 29-38.

Wikström, P. y Sampson, R. (2003). Social mechanisms of community influences on crime and pathways in criminality. En B. Lahey, T. Moffitt, y A. Caspi (eds.), Causes of Conduct Disorder and Serious Juvenile Delinquency (p. 118-148). New York: Guilford Press.

Juan Antonio Rodríguez es Licenciado en Criminología y Doctor en Psicología Social por la Universidad de Santiago de Compostela. Profesor, investigador y ex-director de la Escuela de Criminología de la Universidad de Los Andes en Venezuela. Actualmente es investigador acreditado de la ONCTI y del CDCHTA-ULA (Venezuela). Coordinador del Proyecto ISRD-3 en Venezuela. Sus investigaciones se centran en temas vinculados a la desviación juvenil, el miedo al delito, los métodos cuantitativos en criminología y la criminología comparada. 


\section{ANEXO}

\section{Escala de apoyo parental}

¿Con qué frecuencia tu madre/padre realiza las conductas que aparecen a continuación?

1. Consolarte cuando estás triste

2. Confiar en ti

3. Abrazarte para mostrarte afecto y/o apoyo

4. Ayudarte cuando tienes problemas

5. Decirte que le importas

6. Ayudarte a superar momentos de crisis

7. Decirte algo para hacerte sentir bien

8. Respetar tu individualidad, tu manera de ser

9. Respetar tus sentimientos

10. Escuchar tu punto de vista y hacerte ver que le importas

11. Hablar contigo de temas importantes para ti

12. Apoyarte emocionalmente

Nunca $=0$; Pocas veces $=1$; Algunas veces $=2$; Bastantes veces $=3$; Con mucha frecuencia $=4$

Ítems sobre supervisión parental

1. ¿Tus padres se dan cuenta cuando llegas tarde a casa?*

2. ¿Tus padres saben dónde estás cuando no te encuentras en casa?

3. ¿Cuando estás fuera de casa te comportas de acuerdo con lo que tus padres consideran correcto?

4. ¿Tus padres te piden que les informes dónde y con quién te encuentras mediante llamadas, mensajes de texto, correos o notas?

5. ¿Tus padres conocen a tus amigos? ? $^{* *}$

${ }^{*}$ Nunca $=0 ;$ A veces $=1 ;$ A menudo $=2 ;$ Siempre $=3$

${ }^{* *} \mathrm{~A}$ ninguno $=0 ; \mathrm{A}$ algunos $=1 ; \mathrm{A}$ casi $\operatorname{todos}=2 ; \mathrm{A}$ todos $=3$

Ítems sobre amigos antisociales

¿Cuántos de tus amigos/as que viven en tu comunidad han realizado o les ha sucedido lo que te indicamos a continuación?

1. Destrozar cosas en lugares públicos

2. Llevar armas de fuego/armas blancas

3. Amenazar o atacar a otras personas

4. Discutir violentamente con los padres o los profesores

5. Robar

6. Destrozar cosas de otras personas

7. Ser detenido por la policía 
Ninguno $=0 ;$ Algunos $=1 ; \operatorname{Muchos}=2 ;$ Todos $=3$

Escala de desorden social

¿Qué tan de acuerdo estás con las siguientes afirmaciones con respecto a la comunidad o lugar en el que vives?

1. Hay mucha delincuencia en mi comunidad

2. Hay muchas ventas de droga en mi comunidad

3. Hay muchas peleas en la zona donde vivo

4. En mi comunidad hay mucho consumo de alcohol

5. Hay mucho consumo de drogas en la zona donde vivo

6. Hay mucha venta de alcohol en mi comunidad

7. En la zona donde vivo se escucha música a alto volumen en espacios públicos (parques, calles, plazas, etc.)

Totalmente en desacuerdo=0; En desacuerdo=1; De acuerdo=2; Totalmente de acuerdo $=3$

\section{Escala de eficacia colectiva}

¿Qué tan de acuerdo estás con las siguientes afirmaciones con respecto a la comunidad o lugar en el que vives?

1. Los vecinos están muy unidos en mi comunidad

2. Los vecinos de mi comunidad son de confiar

3. Mis vecinos se dan cuenta cuando me estoy portando mal y me lo hacen saber

Totalmente en desacuerdo=0; En desacuerdo=1; De acuerdo $=2 ;$ Totalmente de acuerdo $=3$

\section{Ítems sobre delincuencia juvenil}

¿Tú has realizado, y con qué frecuencia, las conductas que aparecen a continuación?

1. Darle una golpiza o paliza a alguien

2. Robar cosas de un carro estacionado

3. Entrar a robar en una vivienda

4. Forcejear con alguien para robarle

5. Pegar una patada a alguien

6. Robar cosas de una tienda estando abierta

7. Robar en una vivienda en particular

8. Andar con un arma (navaja, pistola, etc.) por si se necesita en una pelea

9. Robar una moto, bicicleta, para dar una vuelta

10. Atacar a un policía, para impedir que detenga a alguien

11. Convencer a alguien de que haga algo ilegal 
12. Agredir a alguien con intención de matarlo

13. Robar objetos del colegio

14. Ofrecer resistencia a un policía que pretende detenerte

15. Formar parte de un robo utilizando armas

16. Robar el bolso o la cartera de alguien cuando va por la calle

17. Vender drogas

18. Ser detenido por vender drogas

19. Robar cosas de los morrales de tus compañeros de colegio

20. Atracar a alguien

21. Entrar a robar una tienda cerrada

Nunca $=0 ;$ Casi nunca $=1$; Algunas veces $=2$; Casi siempre $=3 ;$ Siempre $=4$ 